\title{
Identification of Partner Proteins of the Algae Klebsormidium nitens NO Synthases: Toward a Better Understanding of NO Signaling in Eukaryotic Photosynthetic Organisms
}

\author{
Pauline Chatelain, Jeremy Astier, David Wendehenne, Claire Rosnoblet and \\ Sylvain Jeandroz*
}

Agroécologie, AgroSup Dijon, CNRS, INRAE, Université Bourgogne Franche-Comté, Dijon, France

OPEN ACCESS

Edited by: Noelia Foresi, CONICET Mar del Plata, Argentina

Reviewed by: Mirko Zaffagnini,

University of Bologna, Italy Koichi Hori,

Tokyo Institute of Technology, Japan

*Correspondence:

Sylvain Jeandroz sylvain.jeandroz@agrosupdijon.fr

Specialty section:

This article was submitted to

Plant Physiology,

a section of the journal

Frontiers in Plant Science

Received: 18 October 2021 Accepted: 30 November 2021 Published: 22 December 2021

Citation:

Chatelain P, Astier J, Wendehenne D, Rosnoblet $C$ and Jeandroz S (2021) Identification of Partner Proteins of the Algae Klebsormidium nitens NO

Synthases: Toward a Better Understanding of NO Signaling in Eukaryotic Photosynthetic Organisms.

Front. Plant Sci. 12:797451 doi: $10.3389 / f p / s .2021 .797451$
In animals, $\mathrm{NO}$ is synthesized from L-arginine by three isoforms of nitric oxide synthase (NOS) enzyme. NO production and effects have also been reported in plants but the identification of its sources, especially the enzymatic ones, remains one of the critical issues in the field. NOS-like activities have been reported, although there are no homologs of mammalian NOS in the land plant genomes sequenced so far. However, several NOS homologs have been found in algal genomes and transcriptomes. A first study has characterized a functional NOS in the chlorophyte Ostreococcus tauri and the presence of NOS homologs was later confirmed in a dozen algae. These results raise the questions of the significance of the presence of NOS and their molecular diversity in algae. We hypothesize that comparisons among protein structures of the two KnNOS, together with the identification of their interacting partner proteins, might allow a better understanding of the molecular diversification and functioning of NOS in different physiological contexts and, more generally, new insights into NO signaling in photosynthetic organisms. We recently identified two NOS homologs sequences in the genome of the streptophyte Klebsormidium nitens, a model alga in the study of plant adaptation to terrestrial life. The first sequence, named KnNOS1, contains canonical NOS signatures while the second, named KnNOS2, presents a large C-ter extension including a globin domain. In order to identify putative candidates for KnNOSs partner proteins, we draw the protein-protein interaction networks of the three human NOS using the BioGRID database and hypothesized on the biological role of $K$. nitens orthologs. Some of these conserved partners are known to be involved in mammalian NOSs regulation and functioning. In parallel, our methodological strategy for the identification of partner proteins of KnNOS1 and KnNOS2 by in vitro pull-down assay is presented.

Keywords: nitric oxide synthase, algae, NO signaling, protein partners, interactome 


\section{INTRODUCTION}

\section{Nitric Oxide and Its Biosynthesis}

$\mathrm{NO}$ is a ubiquitous signaling gaseous molecule which regulates a wide array of physiological, biochemical, and molecular events in animals, plants, and microbes. In mammals, NO is mainly synthetized from L-arginine by three isoforms of nitric oxide synthases (NOSs, EC 1.14.13.39): neuronal NOS (nNOS) or NOS1, inducible NOS (iNOS) or NOS2, and endothelial NOS (eNOS) or NOS3 (Stuehr et al., 2004). Each NOS is a modular enzyme that consists of an N-terminal oxygenase domain and a C-terminal reductase domain, both domains being connected by a short calmodulin binding site (Poulos et al., 1998). In addition to this latter, NOS contains binding sites for NADPH, flavin mononucleotide (FMN), flavin adenine dinucleotide (FAD), tetrahydrobiopterin $\left(\mathrm{BH}_{4}\right)$, and a heme group. Functional NOSs are active as homodimer and transfer electrons from $\mathrm{NADPH}$ to their heme center via FMN and FAD, where L-arginine is oxidized to L-citrulline and NO (Poulos et al., 1998; Wendehenne et al., 2003). Mechanism for NO catalysis is presented in Figure 1. NOS is present in all kingdoms. In metazoans, phylogenetic and syntenic analyses support the view that NOS was recurrently duplicated in different lineages, acquiring new structural configurations through gains and losses of protein motifs (Andreakis et al., 2011).

In plants, NO production and effects have been reported in many physiological processes even if unresolved issue concerns its enzymatic sources (Besson-Bard et al., 2008; Astier et al., 2018; León and Costa-Broseta, 2020). Indeed, we observe a multiplicity of NO sources, from reductive pathways, to oxidative pathways. Besides nitrite-dependent pathway involving nitrate reductase, NOS-like activities, L-arginine-dependent, and sensitive to mammalian NOS inhibitors, have been reported, although there are no homologs of mammalian NOS in the land plant genomes sequenced so far (Astier et al., 2018).

\section{NO in Algae}

Several reports have suggested that algae from different lineages, such as chlorophytes, charophytes, red algae, or diatoms, could produce NO (Mallick et al., 1999, 2000; Sakihama et al., 2002; Tischner et al., 2004; Chung et al., 2008; Vardi et al., 2008; Chow et al., 2013), and a review of the relevant literature indicates that $\mathrm{NO}$ is involved in various functions in algae (Kumar et al., 2015; Astier et al., 2021). Briefly, NO contributes to stress responses, particularly to abiotic ones (e.g., hypoxia, wounding, UV or visible light irradiation, chemicals, heat stress, high concentrations of salt, and metal). NO is also involved in cell physiology (development and metabolism) and could participate in greenhouse gas emission through the reduction of $\mathrm{NO}$ into $\mathrm{N}_{2} \mathrm{O}$ in Chlamydomonas reinhardtii (Burlacot et al., 2020). As already described in land plants, NO can interact with other signaling molecules, such as hydrogen peroxide and calcium for effective cellular signaling as exemplified in the marine macroalga Ulva compressa during the response to copper stress (González et al., 2012).

NOS-like activity has been detected using NOS inhibitors in various marine algae, such as Ulva compressa (Chorophytes), Chattonella marina (Ochrophyta), or Symbiodinium microadriaticum (Dinoflagellates; Kumar et al., 2015), raising

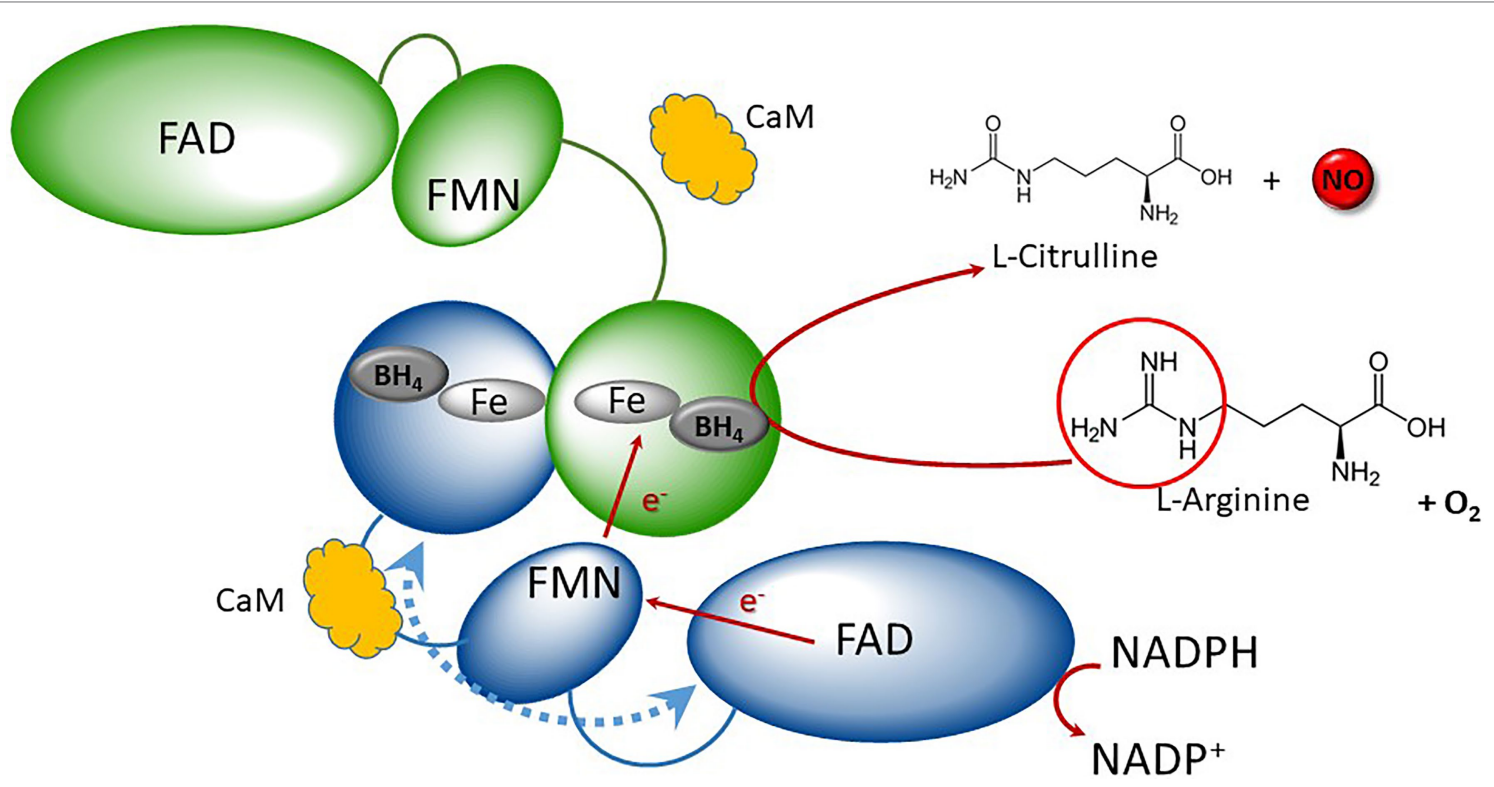

FIGURE 1 | Schematic representation of canonical NOS catalytic mechanism. Canonical NOSs display homodimeric quaternary structure (blue and green monomers). NOS monomers consist of two main domains: the N-terminal oxygenase (round-shaped) and the C-terminal reductase, containing FNR (ellipse) and flavodoxin (small ellipse). They both shelter different redox cofactors: FAD, FMN, heme, and BH4. Reductase and oxygenase domains are interconnected by a Calmodulin (CaM, orange cloud)-binding domain. Electrons are purveyed by NADPH and are shuttled to the flavodoxin via the FAD. Upon Cam binding, electron transfer then occurs from the FMN of one monomer (blue) to the heme of the second one (green), resulting to the oxidation of Arginine in presence of oxygen into Citrulline + NO. Adapted from Santolini (2019). 
the question of the presence of NOS. A first study conducted by Foresi et al. (2010) characterized one functional NOS in the chlorophyte Ostreococcus tauri (see also Weisslocker-Schaetzel et al., 2017). In the last decade, massive genome and transcriptome sequencing in plants have revealed several NOS homologs in algae (Jeandroz et al., 2016; Santolini et al., 2017). These NOS are distributed in different phylogenetic groups, in the green lineage but also in the more distant diatoms and dinoflagellates. As described in an exhaustive way by Santolini (2019), the diversity of NOS proteins resides not only in the organismal diversity in which these genes/proteins could be found but also in the number and types of NOSs that are present in any organisms. Indeed, besides the "archetypal" or "standard" NOS, firstly defined in mammals, we now have to consider several different groups of NOSs, corresponding to different architectural types of proteins and perhaps different functions.

Taken together, these results raise questions: why did NOS homologs disappear in land plants during evolution? Why NOS homologs remained in algae and what is the meaning of the NOS molecular diversity in photosynthetic organisms? The characterization and functional analyses of these algal NOSs could provide first answers to these questions.

\section{WORKING HYPOTHESIS: NO SYNTHASES INTERACT WITH PARTNER PROTEINS THAT COULD MODULATE THEIR ACTIVITIES AND SPECIFICITIES}

Protein-protein interactions (PPI) are the crucial events in cellular signaling mechanisms. In mammals, the three identified NOS isoforms display specific profiles of expression, cellular/ subcellular localization, regulation, or catalytic properties. Emerging evidence indicates a role for PPI as a regulator of NOS activity and therefore specificity. For instance, destabilization of the active NOS dimeric structure or preventing its dimerization by PPI leads to its inactivation (Zhou and Zhu, 2009; Cinelli et al., 2020) while NOS activity (NO production) can be optimized by a partner protein which facilitates the delivery of its substrate (Kone, 2000). Moreover, specific interaction of the PDZ N-ter domain with the dystrophin complex is responsible for human NOS1 subcellular distribution (Brenman et al., 1995). PPI are also at the origin of multiple component signaling modules, associating NOS, scaffolding, and S-nitrosated protein. For instance, the NOS2 interaction with the protein PSD95, leading to the regulation of the NMDA receptor by S-nitrosylation, constitutes a noteworthy illustration of the spatiotemporal aspect of NO signaling (Stamler et al., 2001).

We hypothesize that comparisons among protein structures of the NOS, together with the identification of their partner proteins, might allow a better understanding of the molecular diversification and functioning of NOS in different physiological contexts and, more generally, new insights into NO signaling in photosynthetic organisms.

In order to test this hypothesis, we use the charophyte (streptophytes) Klebsormidium nitens as a biological model to investigate these NOSs and their structural and functional diversity. K. nitens presents two interests. Firstly, its complete genome sequence has revealed several keys signaling modules, known to interact with NO in land plants (e.g., phytohormones) and required for plant terrestrial adaptation (Hori et al., 2014). Secondly, BLAST searches using OtNOS revealed the presence of two protein sequences showing specific NOS molecular patterns but different architectures (domain organization), which makes it possible to address the question of the structure/ activity relationships of NOS.

\section{MOLECULAR DIVERSITY OF KnNO SYNTHASES AND THEIR RELATIVES IN ALGAE}

\section{Two Putative KnNOSs Are Found in the K. nitens Genome KnNOS1, The "Standard" Model}

This protein of 1099 aa (GAQ81306.1) shares 36\% of identity with OtNOS, the first NOS characterized in algae (Foresi et al., 2010). KnNOS1 possesses classical mammalian NOS architecture consisting of oxygenase and reductase domains (Figure 2A). Globally, KnNOS1 structure is close to the other NOSs described in green algae (Santolini et al., 2017). Compared to mammalian NOSs, these algal NOSs lack the key conserved residues of the $\mathrm{N}$-ter hook as well as those involved in binding the dihydroxypropyl side chain of $\mathrm{BH}_{4}$. A structural analysis of the NOSoxy domain shows that algal NOSs conserve the key structural features of mammalian, including the proximal heme ligand Cys, the L-Arg- and $\mathrm{BH}_{4}$-binding residues, and a modified helical lariat and helical $\mathrm{T}$ region that are involved in binding the pterin cofactor and in the interactions between monomers that help stabilize the homodimer (Figure 2B). They also possess an atypical putative zinc-binding region (Cys-X3-Cys instead of the typical Cys-X4-Cys; Santolini et al., 2017).

\section{KnNOS2, The "NOS-Globin" Model}

we identified in the $K$. nitens genome a second putative NOS sequence showing a large C-ter extension of approximately 380 residues (GAQ90485.1). Conserved protein domain analysis using CD-Search in NCBI (Marchler-Bauer et al., 2011) reveals an ANK motif and a globin domain. The globin domain belongs to the $\mathrm{M}$ family globin domain and shows a characteristic $\alpha$-helical secondary structure with two distal and proximal His residues involved in the coordination of heme-Fe (Vázquez-Limón et al., 2012). The combination of a globin domain to a NOS protein in the C-ter end has already been identified by Santolini (2019) in the green algae Gonium pectorale (Chlorophytes; Figure 3). Interestingly, we recently identified other NOS proteins showing the chimeric NOS-Globin organization in two other charophytes (Chlorokybus atmophyticus and Mesostigma viride) and three chlorophytes Chlamydomonas incerta, C. schloessori et Edaphochlamys debaryana (Figures 2, 3). This NOS-Globin organization seems to be evolutionary significant as it appears in three of the six major clades of charophytes, the earliest 


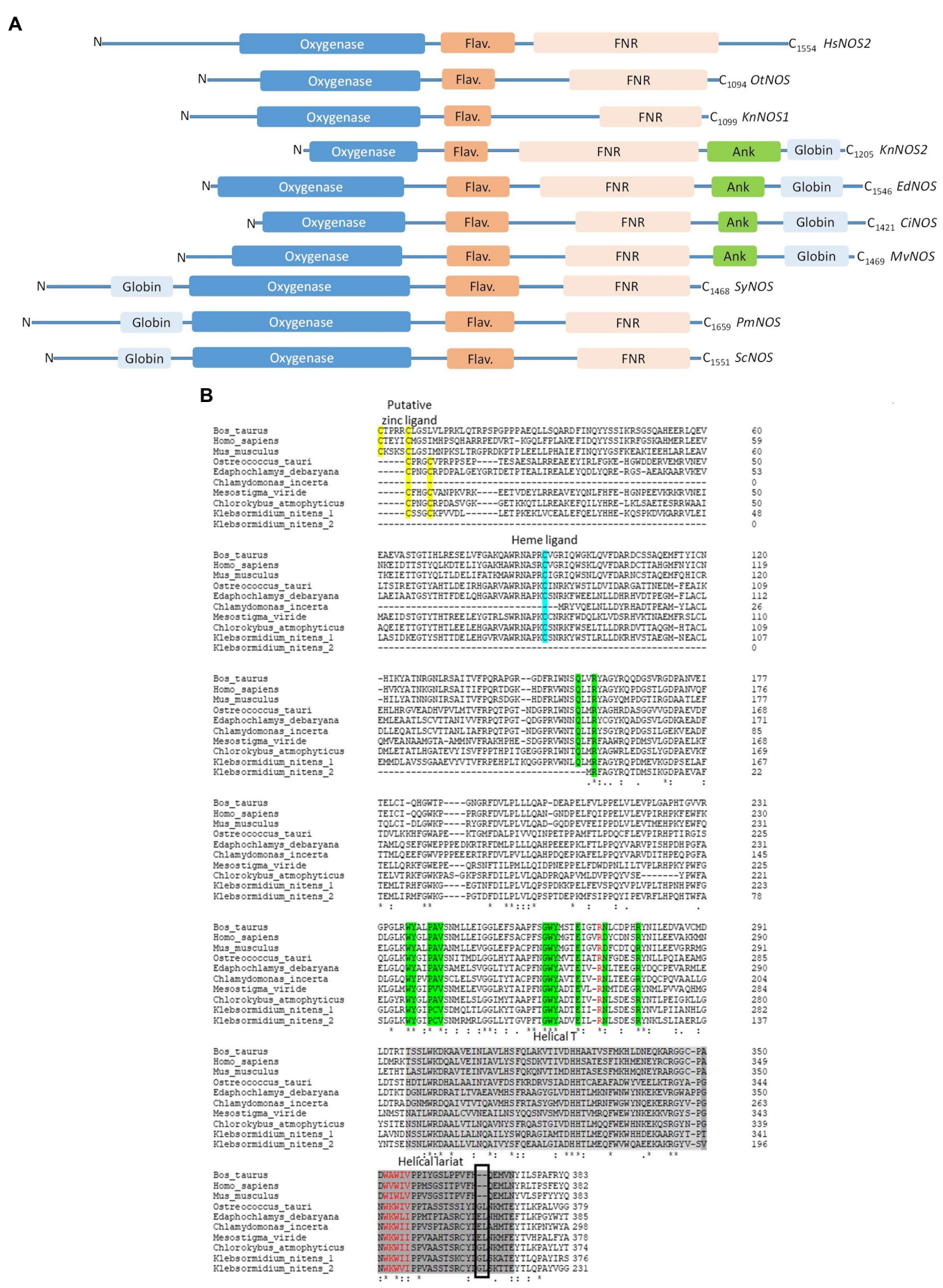

FIGURE 2 | (A) Schematic representation of primary sequences of NOS-like proteins from Homo sapiens (HsNOS2, AAB60654), Ostreococcus tauri (OtNOS, OUS45267), Klebsormidium nitens (KnNOS1, KFL_000760350 at the top and KnNOS2, KFL_006460015 at the bottom), Edaphochlamys debaryana (EdNOS, KAG2495438), Chlamydomonas incerta (CiNOS, KAG2438015), Mesostigma viride (MvNOS, Mv7497-RA-2, Joint Genome Institute PhytoCosm), Synechococcus sp. (SyNOS, WP_006458277), Pseudo-nitzschia multistriata (PmNOS), and Skeletonema costatum (ScNOS). Three NOSs share a common oxygenase domain (blue). The FMN domain is represented by a dark orange flavodoxin module (Flav.) and the FAD/NADPH domain is represented by a light orange FNR box. Additional domains comprise a globin domain (light blue box), an ankyrin-repeat domain (Ank, green). Conserved domains have been identified thanks to NCBI and domains alignment analyzed through Clustal Omega. (B) Comparative analysis of NOS oxygenase domains from Bos Taurus (AAA30669.1), Homo sapiens (HsNOS2, AAB60654), Mus musculus (AAA39834.1), Ostreococcus tauri (OUS45267), Edaphochlamys debaryana (KAG2495438), Chlamydomonas incerta (KAG2438015), Mesostigma viride (MvNOS, Mv7497-RA-2, Joint Genome Institute PhytoCosm), Chlorokybus atmophyticus (CaNOS, chrsp63S00556, Joint Genome Institute PhytoCosm), and K. nitens (1, KFL_000760350 and 2, KFL_006460015). Arginine binding residues are colored in green. The black box highlights the insert observed in the helical lariat of non-mammalian NOSs. Alignment has been performed through Clustal Omega. 


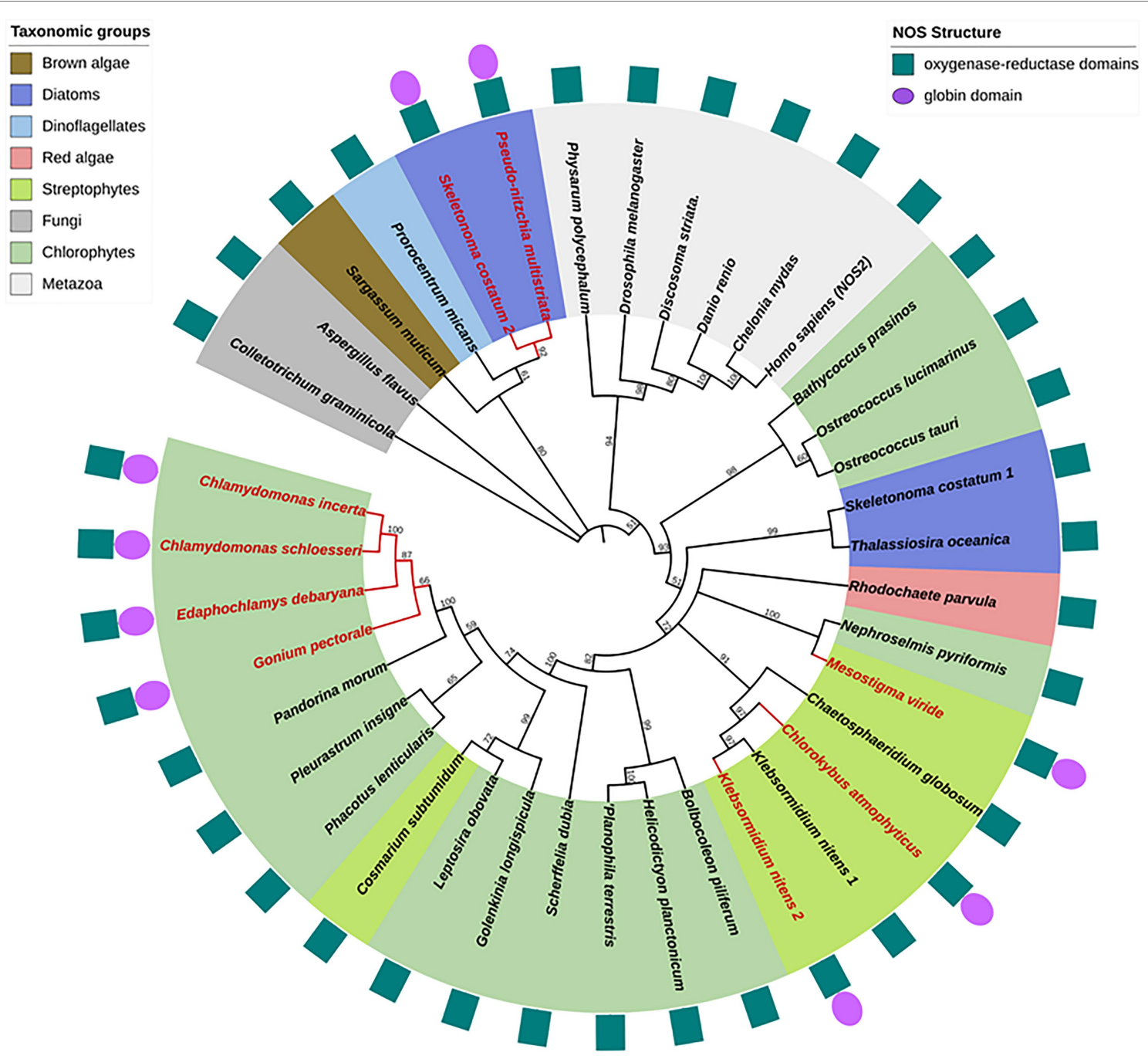

FIGURE 3 | Phylogenetic reconstruction of NOS proteins. Algal NOS sequences were extracted from databases (see Santolini et al., 2017). NOS proteins were aligned with ClustalW and the phylogenetic tree was constructed by maximum likelihood method using MEGA (Tamura et al., 2021). Bootstrap analysis was carried out using 500 replicates. All nodes with bootstrap values less than $50 \%$ were collapsed into polytomies. Branches and labels in red indicate chimeric structures. Protein domain organization is depicted around the phylogenetic tree.

diverging lineages of extant streptophytes. Biological functions of these proteins need to be studied to validate their role in algal physiology. The association of globin and NOS domains has been firstly characterized in the free-living marine unicellular cyanobacterium Synechococcus PCC 7335 (Correa-Aragunde et al., 2018), but in this protein, the globin domain is located at the N-ter of the protein (Globin-NOS). Biochemical analysis showed that SyNOS produces $\mathrm{NO}$ and $\mathrm{NO}_{3}{ }^{-}$in vitro and data suggest that the globin domain is mainly responsible for $\mathrm{NO}_{3}{ }^{-}$ production (Picciano and Crane, 2019). It is proposed that the globin domain in SyNOS could confer new functional capabilities for $\mathrm{N}$ metabolism (Correa-Aragunde et al., 2018). Interestingly, chimeric globin-NOSs similar to SyNOS appear in some diatom genomes, such as Pseudo-nitzschia multistriata (PmNOS) and Skeletonema costatum (ScNOS; Di Dato et al., 2015; Figure 2A).

\section{Phylogenetic Diversity of NO Synthases in Photosynthetic Organisms}

As previously reported, algal NOSs are unequally distributed in the photosynthetic organism phylogeny (Jeandroz et al., 2016; Santolini et al., 2017). They were found in the green lineage (streptophytes and chlorophytes) but also in red algae, diatoms, dinoflagellates, and brown algae (Figure 3). Several discrepancies between the standard plant phylogeny and the one deduced from NOS proteins are noticed, indicating duplication/loss events or horizontal gene transfers along NOS gene evolution and consequently putative functional homoplasy. For examples, chlorophytes NOSs are not grouped together and the green lineage (streptophytes and chlorophytes) is not, contrary to plant phylogeny, a monophyletic group since several diatoms and red algae NOS belong to the same clade. Interestingly, both KnNOSs are grouped 
together, and KnNOS2 is not related to other NOS-Globin found in E. debaryana and in the two Chlamydomonas species. This indicates that the NOS-globin chimeric organization, found in seven species, could have independent origins. The Globin-NOS organization, firstly characterized in the cyanobacterium Synechococcus PCC 7335, is restricted to the two diatoms $P$. multistriata and S. costatum. Noticeably, this latter species harbors a second NOS, with a classical organization, which is distantly related from the NOS-Globin in the phylogenetic tree.

\section{PUTATIVE NO SIGNALING ACTORS IN $K$. nitens GENOME SEQUENCE}

In mammals, the biological effects of NO are mediated notably via a NO/cGMP depending cascade which requires the mobilization of soluble guanylyl cyclases (sGCs), cGMP, cGMPdependent protein kinases (PKGs), and cyclic nucleotide phosphodiesterases (PDEs; Martínez-Ruiz et al., 2011). We recently investigated the presence of these major NO-signaling cascade components in the green lineage (Astier et al., 2019). None of the +1000 species tested harbored all the required enzymes necessary for the classical NO/cGMP depending cascade. However, a few homologs of soluble guanylate cyclases, cGMP-dependent protein kinases, cyclic nucleotide-gated channels, and cGMP-regulated PDEs were identified punctually in some of the organisms tested, mainly algae.

Interestingly, among the candidates identified, some were further characterized, such as the sGCs CYG12, CYG56, and CYG11 from C. reinhardtii. CYG12 protein shares 40 to $50 \%$ identity with animal GCs and an initial structural analysis demonstrated that its conformation allows a functional cyclase activity (Winger et al., 2008). Recently, the enzyme was demonstrated to be functional and to participate in hypoxia responses in C. reinhardtii (Düner et al., 2018). Interestingly, $\mathrm{NO}$ is also involved in hypoxia responses (Gupta et al., 2020; Manrique-Gil et al., 2021). However, in vitro approaches using NO donors on the recombinant protein failed to demonstrate an increase of the enzymatic activity (Düner et al., 2018). Therefore, in vivo approaches are required to further confirm or invalidate the involvement of CYG12 in a NO/cGMP signaling cascade. Another guanylate cyclase, CYG56, was found to be involved in the regulation of nitrate assimilation, through its participation in the NO/cGMP-dependent signaling cascade mediating nitrate reductase repression induced by ammonium (de Montaigu et al., 2010). However, similarly to CYG12, CYG56 cyclase activity did not show NO sensitivity in vitro. Concerning CYG11, the protein displays unique features as compared to other described eukaryotic guanylate cyclases (Horst et al., 2019). Structurally, this enzyme possesses two HNOX domains and is active as a homodimer. Interestingly, its activity is increased upon carbon monoxide (CO) exposure, rather than NO exposure. Based on its enzymatic parameters, $\mathrm{CO}$ may represent the physiological ligand of CYG11, making this enzyme a CO-sensing protein in C. reinhardtii, although the confirmation of this hypothesis will require further investigations. The characterization of these three guanylate cyclases demonstrates the importance of cGMP-dependent signaling in algae, although a direct link with NO-dependent signaling remains to be clearly established.

The first proper biochemical characterization of a plant cGMP/ cAMP-dependent protein kinase was only reported recently in rice (Shen et al., 2019). Presenting atypical features compared to animal ones, notably the coexistence of a phosphatase and a kinase domain within the sequence, this PKG was found to be involved in gibberellin (GA) signaling and salt stress responses, two pathways where $\mathrm{NO}$ acts as a signaling molecule. Nevertheless, the corresponding molecular actors, particularly those related to NO and cGMP synthesis, remain to be identified to confirm the existence of a GA/NO/cGMP signaling cascade in rice. Interestingly, homologs of this rice $\mathrm{PKG}$ are generally present in single copy and well distributed among the green lineage, in algae and land plants. Concerning the cGMP turnover, the presence of PDEs related to NO signaling in the green lineage is yet to be demonstrated. A unique description of a putative class I PDE has been reported in C. reinhardtii (Gross and Durner, 2016), but its activity is yet to be characterized. Additionally, the first functional cGMP-activated PDE from plants was recently described in Arabidopsis thaliana (Isner et al., 2019). However, here again, no information about the NO-dependency of this protein activity, related to UV-A responses, has been reported.

Similarly, the relation between NO and CNGCs is not fully elucidated in plants. Out of our bioinformatic analyses, no NO-signaling-related CNGC homologs were found in the green lineage (Astier et al., 2019). Accordingly, no regulation of plant or algae CNGCs by NO-dependent mechanisms has been reported so far.

A closer search on the putative NO-signaling modules that can be found in $K$. nitens revealed the presence of some candidates. If no homologs of animal type NO-dependent sGC of CNGCs could be retrieved, the search for protein homologs of the putative CrPDE (XP_001689825.1) and rice PKG (Q6K3D4_ ORYSJ), with a minimum of $30 \%$ identity on $60 \%$ of the query length, leads to the identification of, respectively, 3 (GAQ77586.1, GAQ86043.1, and GAQ84593.1) and 1 (GAQ83023.1) candidates. The determination of their respective roles in K. nitens together with their potential link with NO signaling and KnNOSs activities constitutes a promising ax of investigation.

\section{IDENTIFICATION AND ROLE OF NOSS INTERACTING PARTNER PROTEINS}

In order to identify putative candidates for putative KnNOSs partner proteins, we first draw the PPI network of the three human NOS isoforms using the BioGRID database, which congregates PPI data based on experimental approaches (Figure 4). This network reconstruction indicates specific interactions between each of the three isoforms with privileged partners. NOS2 presents the largest numbers of interactors with 175 nodes, compared to NOS1 and NOS3 with 16 and 46 nodes, respectively. Some partners interact with more than one human NOS and allow the interconnection of the three subnetworks. Each of the three NOS interacts with itself, as suggested by the active dimeric form of NOS (Venema et al., 1997). 


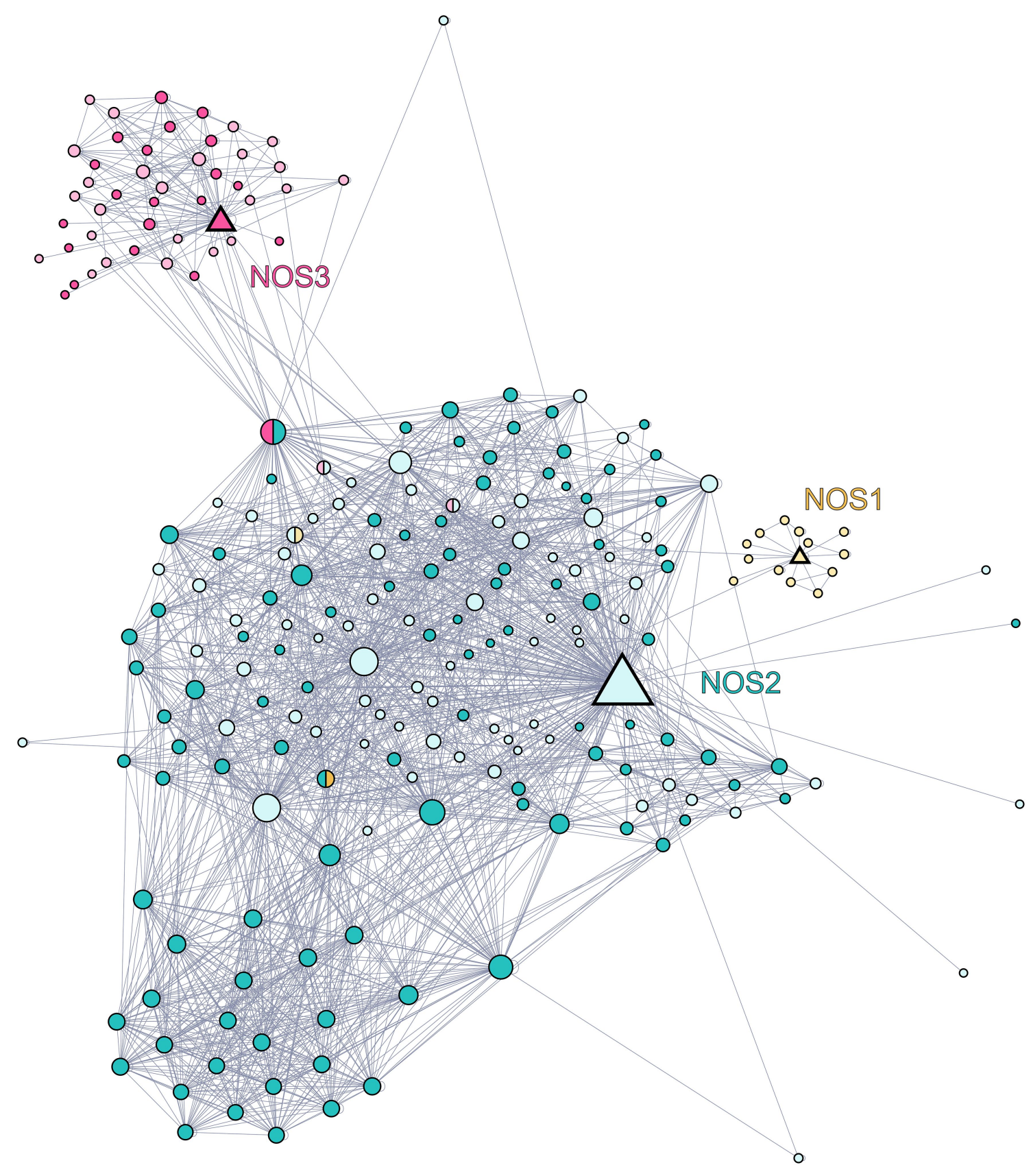

FIGURE 4 | Network of the protein-protein interaction network of human NOS1 (neuronal NOS; P29475), NOS2 (inducible NOS; P35228), and NOS3 (endothelial NOS; P29474). Data were downloaded from the BioGRID database and graphical representation was carried out using Gephi software. Each node represents a protein, and the size of the nodes is proportional to the number of connections with other nodes. The links between nodes represent physical interactions between proteins. The triangle-shaped nodes represent the three NOSs, yellow for NOS1, blue for NOS2, and red for NOS3. The circles-shaped nodes represented in blue are partners of NOS2, those in red are partners of NOS3, and those in yellow are partners of NOS1. Nodes represented in pastel shade do not have orthologs in $K$. nitens while those in dark colors are conserved in $K$. nitens.

Starting from this confirmed NOS interacting protein list identified in humans, we then look for the presence of their orthologs in $K$. nitens. We developed an in silico approach using OrthoFinder, a powerful tool which allows whole-genome comparisons (Emms and Kelly, 2019). Among partner proteins of human NOS1, NOS2, and NOS3, 6.25,
58.3 , and $45.8 \%$ are conserved in the proteome of $K$. nitens, respectively. These conserved proteins are represented in dark shades in PPI network (Figure 4). Since orthologous proteins are more likely to share the same function, we are able to hypothesize on the biological role of $K$. nitens orthologs (Emms and Kelly, 2015). Some of these conserved 
partners are known to be involved in mammalian NOSs regulation and functioning. Several interesting candidates were identified and detailed below.

\section{Control of NOS Activity: The Role of HSP90 and Actin}

NOS activity can be enhanced by formation of a ternary complex with HSP90 and globular $\beta$-actin (Table 1). Firstly, HSP90 binds to NOS which results in an increase in NOS activity as well as an increase in affinity for $\beta$-actin. Secondly, binding of $\beta$-actin results in an increase of HSP90 degradation, and in the dissociation of the ternary complex, thus limiting the time in which NOS3 is active. This mechanism increases the production of NO when the globular form of $\beta$-actin is favored over the fibrillar form while maintaining a negative feedback (Ji et al., 2007).

\section{Proteasomal Degradation of NOS: The Role of STUB, Elongin C, ADRM1, and UCH37}

Binding of partners with NOS could trigger the proteasomal degradation of the relevant NOS isoform and could constitute another level of regulation (Table 1). For example, in human cells, it has been shown that STUB1, via its ubiquitin ligase activity triggers the degradation of NOS1 by ubiquitinating the calmodulin binding site (Clapp et al., 2012). A similar mechanism has been described for Elongin $\mathrm{C}$ which interacts and ubiquitinylates NOS2. This ubiquitin motif is then recognized by the $19 \mathrm{~S}$ proteasome subunit ADRM1, followed by the recruitment of $\mathrm{UCH} 37$, leading to proteasomal degradation of NOS2 (Mazumdar et al., 2010; Nishiya et al., 2011).

\section{Changes of NOSs Intracellular Distribution: The Role of Rac2 and NOSIP}

Isoform-specific regulators of NOS intracellular distribution have been identified (Table 1). In the case of NOS2, interaction with Rac2 during inflammatory response leads to an increase of NOS activity through spatial redistribution within the cell (Kuncewicz et al., 2001). In the case of NOS3, the binding of NOSIP in C-terminal domain negatively modulates NOS activity by uncoupling NOS3 from plasma membrane caveolae (Dedio et al., 2001).

Thereby, our preliminary in silico approach highlights the promising presence of two NOSs and putative numerous NOS interacting partners in the genome of K. nitens. We hypothesize that these enzymes would participate in a complex signaling networks, as the ones described in animals, and we seek to determine its specificities and roles.

\section{Identification and Functional Analysis of KnNOS Partner Proteins}

Our methodological strategy for the identification of partner proteins is presented in Figure 5.

The first step will be to produce KnNOS1 and KnNOS2 proteins in heterologous bacterial system (Figure 5A). Once the two tagged recombinant proteins produced by bacteria, purified and then fixed on beads by affinity for the tag, in vitro pull-downs will be carried out through incubations with native extracts of algae proteins. Partners pulled-down through tagged KnNOSs will be then identified by nanoliquid chromatography tandem mass spectrometry analysis (Figure 5B). Negative control will be performed using protein extracts incubated with the tag alone fixed on beads. The list of identified protein partners will be compared to the one obtained with the in silico analysis in order to choose the most promising candidates.

The second step will be a functional analysis of the protein partners. It will consist in genetic approaches implying the generation of knock-out and overexpressing $K$. nitens mutant lines for several candidate partners (Figure 5C). Different methods of transformation could be tested. The electroporation method is operational in several algal strains and particularly in charophytes (Kawai et al., 2021). If unsuccessful, we will test an Agrobacterium tumefaciens-based approach. This latter was efficiently adapted for transformation of microalgae (Doron et al., 2016). The functional consequences of these mutations will be based on the analysis of (1) NO production by fluorimetry with DAF-2DA, (2) cellular morphology by microscopy, and (3) algae responses to abiotic stress.

TABLE 1 | List of human NOSs partners identified from the BioGRID database and their orthologs in Klebsormidium nitens genome.

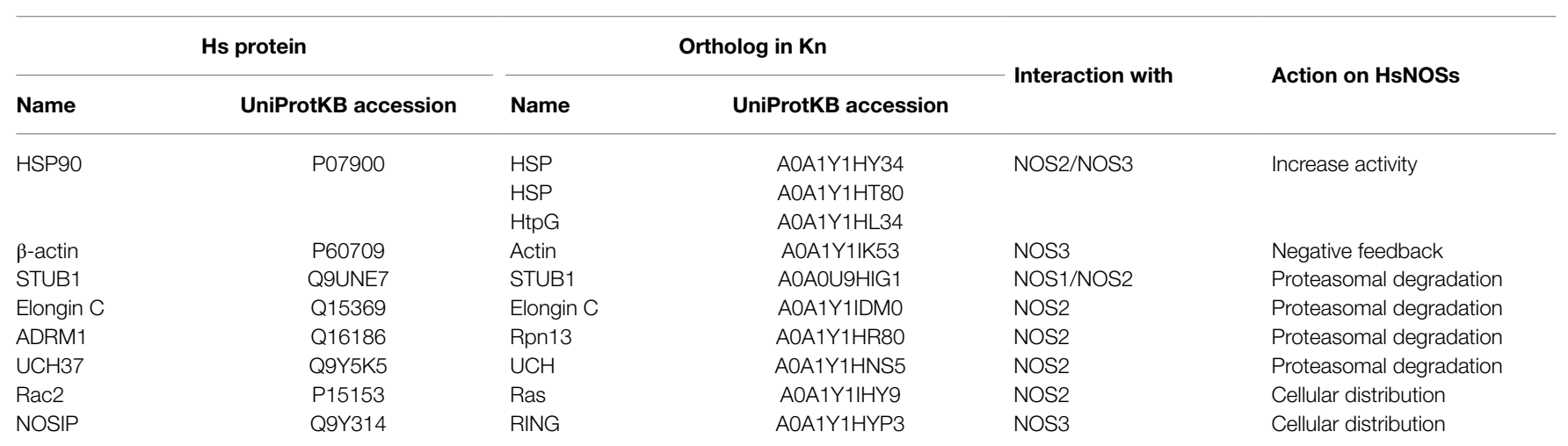




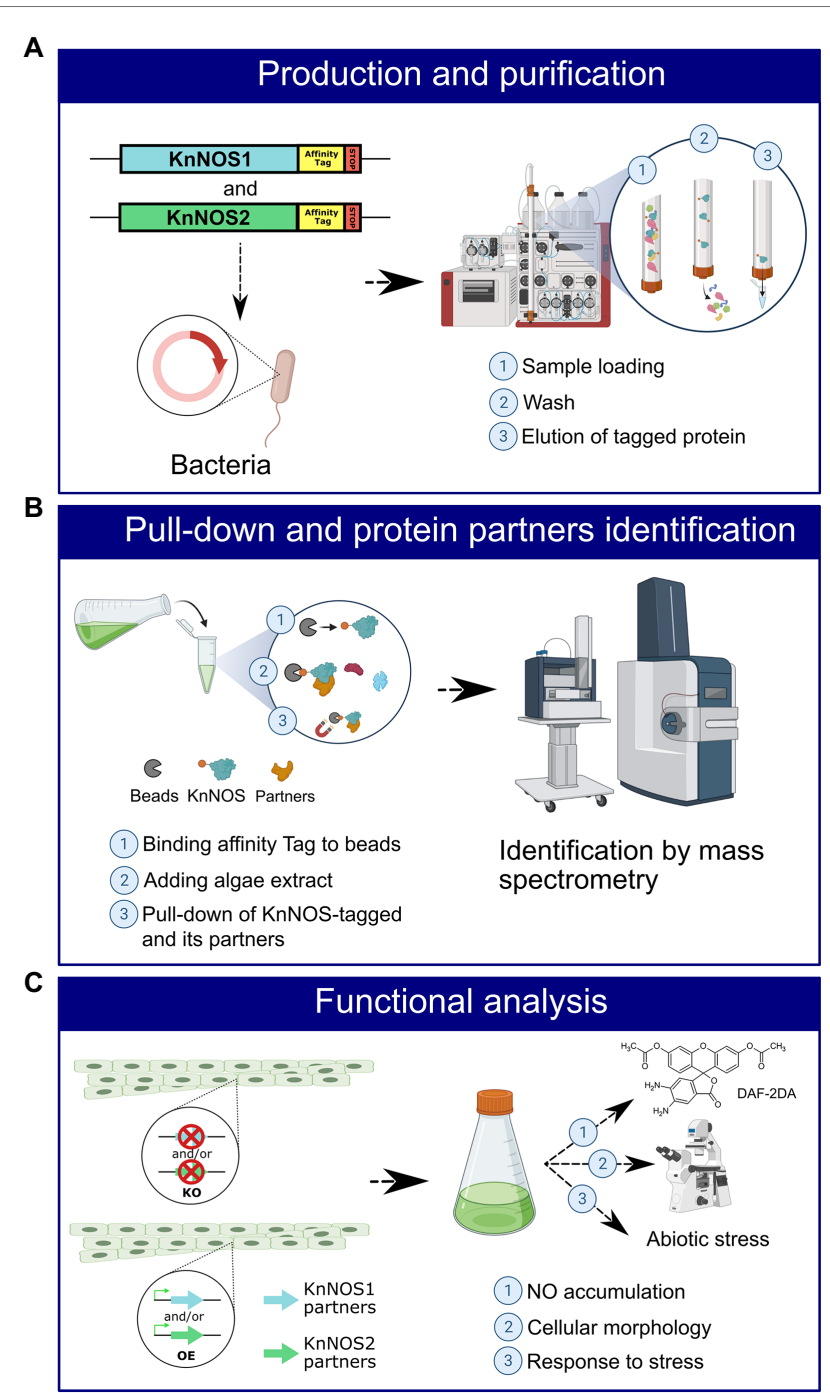

FIGURE 5 | Schematic representation of the strategy for identifying NOSs protein partners and their characterization. (A) An affinity tag will be added by molecular engineering to the sequence coding for KnNOS1 and KnNOS2. The two inserts will be cloned in vector, and the two recombinant proteins will be produced in heterologous system. Recombinant proteins KnNOS1 and KnNOS2 will be purified by affinity chromatography. (B) Purified KnNOS1 and KnNOS2 will be coated on magnetic beads. Pull-down assay will be carried out to precipitate KnNOSs and their respective partner proteins. Partner proteins will be identified by mass spectrometry. (C) A functional analysis using targeted genetic approaches will allow the characterization of the physiological functions of KnNOSs through study of their partner proteins.

\section{REFERENCES}

Andreakis, N., D’Aniello, S., Albalat, R., Patti, F. P., Garcia-Fernàndez, J., Procaccini, G., et al. (2011). Evolution of the nitric oxide synthase family in metazoans. Mol. Biol. Evol. 28, 163-179. doi: 10.1093/molbev/msq179

Astier, J., Gross, I., and Durner, J. (2018). Nitric oxide production in plants: an update. J. Exp. Bot. 69, 3401-3411. doi: 10.1093/jxb/erx420

Astier, J., Mounier, A., Santolini, J., Jeandroz, S., and Wendehenne, D. (2019). The evolution of nitric oxide signalling diverges between animal and green lineages. J. Exp. Bot. 70, 4355-4364. doi: 10.1093/jxb/erz088

\section{CONCLUSION}

The discovery of two NOS isoforms in the K. nitens genome confirms the already observed molecular diversity of these proteins, illustrated by intra-domain amino-acid polymorphisms as well as recombination of protein domains. This diversity reflects the complex evolution of NO signaling in photosynthetic organisms. A first analysis, conducted in silico, has confirmed the presence of putative partner proteins of NOS in the $K$. nitens genome. Now, the use of in vitro assays followed by functional characterization of candidate proteins, in different physiological contexts, will certainly help us to improve our understanding on NOSs regulation and their roles in algae.

\section{DATA AVAILABILITY STATEMENT}

The original contributions presented in the study are included in the article/supplementary material, and further inquiries can be directed to the corresponding author.

\section{AUTHOR CONTRIBUTIONS}

PC, JA, and SJ conducted the in silico analyses. SJ, CR, and DW conceived the study. All authors wrote and edited the manuscript.

\section{FUNDING}

Work in our lab is currently supported by the Ministère de l'Enseignement supérieur, de la Recherche et de l'Innovation, Investissements d'Avenir program, project ISITE-BFC NOISELESS (contract ANR-15-IDEX-0003; grant NOISELESS-RA18041.AEC.IS) and the Agence Nationale de la Recherche, project ALGAE-NOS (grant ANR-18CE20-0022-02).

\section{ACKNOWLEDGMENTS}

We thank Arnaud Mounier for helping on OrthoFinder analysis.

Astier, J., Rossi, J., Chatelain, P., Klinguer, A., Besson-Bard, A., Rosnoblet, C., et al. (2021). Nitric oxide production and signalling in algae. J. Exp. Bot. 72, 781-792. doi: 10.1093/jxb/eraa421

Besson-Bard, A., Pugin, A., and Wendehenne, D. (2008). New insights into nitric oxide signaling in plants. Annu. Rev. Plant Biol. 59, 21-39. doi: 10.1146/ annurev.arplant.59.032607.092830

Brenman, J. E., Chao, D. S., Xia, H., Aldape, K., and Bredt, D. S. (1995). Nitric oxide synthase complexed with dystrophin and absent from skeletal muscle sarcolemma in Duchenne muscular dystrophy. Cell 82, 743-752. doi: 10.1016/0092-8674(95)90471-9 
Burlacot, A., Richaud, P., Gosset, A., Li-Beisson, Y., and Peltier, G. (2020). Algal photosynthesis converts nitric oxide into nitrous oxide. Proc. Natl. Acad. Sci. 117, 2704-2709. doi: 10.1073/pnas.1915276117

Chow, F., Pedersén, M., and Oliveira, M. C. (2013). Modulation of nitrate reductase activity by photosynthetic electron transport chain and nitric oxide balance in the red macroalga Gracilaria chilensis (Gracilariales, Rhodophyta). J. Appl. Phycol. 25, 1847-1853. doi: 10.1007/s10811-013-0005-8

Chung, C.-C., Hwang, S.-P. L., and Chang, J. (2008). Nitric oxide as a signaling factor to Upregulate the death-specific protein in a marine diatom, Skeletonema costatum, during blockage of electron flow in photosynthesis. Appl. Environ. Microbiol. 74, 6521-6527. doi: 10.1128/AEM.01481-08

Cinelli, M. A., Do, H. T., Miley, G. P., and Silverman, R. B. (2020). Inducible nitric oxide synthase: regulation, structure, and inhibition. Med. Res. Rev. 40, 158-189. doi: 10.1002/med.21599

Clapp, K. M., Peng, H.-M., Jenkins, G. J., Ford, M. J., Morishima, Y., Lau, M., et al. (2012). Ubiquitination of neuronal nitric-oxide synthase in the Calmodulin-binding site triggers proteasomal degradation of the protein. J. Biol. Chem. 287, 42601-42610. doi: 10.1074/jbc.M112.412494

Correa-Aragunde, N., Foresi, N., Del Castello, F., and Lamattina, L. (2018). A singular nitric oxide synthase with a globin domain found in Synechococcus PCC 7335 mobilizes N from arginine to nitrate. Sci. Rep. 8:12505. doi: 10.1038/s41598-018-30889-6

de Montaigu, A., Sanz-Luque, E., Galván, A., and Fernández, E. (2010). A soluble Guanylate Cyclase mediates negative signaling by ammonium on expression of nitrate Reductase in Chlamydomonas. Plant Cell 22, 1532-1548. doi: $10.1105 /$ tpc. 108.062380

Dedio, J., König, P., Wohlfart, P., Schroeder, C., Kummer, W., and Müller-Esterl, W. (2001). NOSIP, a novel modulator of endothelial nitric oxide synthase activity. FASEB J. 15, 79-89. doi: 10.1096/f.00-0078com

Di Dato, V., Musacchia, F., Petrosino, G., Patil, S., Montresor, M., Sanges, R., et al. (2015). Transcriptome sequencing of three pseudo-nitzschia species reveals comparable gene sets and the presence of nitric oxide synthase genes in diatoms. Sci. Rep. 5:12329. doi: 10.1038/srep12329

Doron, L., Segal, N., and Shapira, M. (2016). Transgene expression in microalgaeFrom tools to applications. Front. Plant Sci. 7:505. doi: 10.3389/fpls.2016.00505

Düner, M., Lambertz, J., Mügge, C., and Hemschemeier, A. (2018). The soluble guanylate cyclase CYG12 is required for the acclimation to hypoxia and trophic regimes in Chlamydomonas reinhardtii. Plant J. 93, 311-337. doi: 10.1111/tpj.13779

Emms, D. M., and Kelly, S. (2015). OrthoFinder: solving fundamental biases in whole genome comparisons dramatically improves orthogroup inference accuracy. Genome Biol. 16:157. doi: 10.1186/s13059-015-0721-2

Emms, D. M., and Kelly, S. (2019). OrthoFinder: phylogenetic orthology inference for comparative genomics. Genome Biol. 20, 238. doi: 10.1186/s13059019-1832-y

Foresi, N., Correa-Aragunde, N., Parisi, G., Caló, G., Salerno, G., and Lamattina, L. (2010). Characterization of a nitric oxide synthase from the plant kingdom: NO generation from the green alga Ostreococcus tauri is light irradiance and growth phase dependent. Plant Cell 22, 3816-3830. doi: 10.1105/ tpc. 109.073510

González, A., Cabrera, M., de Los, Á., Henríquez, M. J., Contreras, R. A., Morales, B., et al. (2012). Cross talk among calcium, hydrogen peroxide, and nitric oxide and activation of gene expression involving Calmodulins and calcium-dependent protein kinases in Ulva compressa exposed to copper excess. Plant Physiol. 158, 1451-1462. doi: 10.1104/pp.111.191759

Gross, I., and Durner, J. (2016). In search of enzymes with a role in $3^{\prime}$, $5^{\prime}$-cyclic Guanosine monophosphate metabolism in plants. Front. Plant Sci. 7:576. doi: 10.3389/fpls.2016.00576

Gupta, K. J., Kolbert, Z., Durner, J., Lindermayr, C., Corpas, F. J., Brouquisse, R., et al. (2020). Regulating the regulator: nitric oxide control of post-translational modifications. New Phytol. 227, 1319-1325. doi: 10.1111/nph.16622

Hori, K., Maruyama, F., Fujisawa, T., Togashi, T., Yamamoto, N., Seo, M., et al. (2014). Klebsormidium flaccidum genome reveals primary factors for plant terrestrial adaptation. Nature Comm. 5, 3978-3979. doi: 10.1038/ ncomms 4978

Horst, B. G., Stewart, E. M., Nazarian, A. A., and Marletta, M. A. (2019). Characterization of a carbon monoxide-activated soluble Guanylate Cyclase from Chlamydomonas reinhardtii. Biochemistry 58, 2250-2259. doi: 10.1021/ acs.biochem. $9 \mathrm{~b} 00190$
Isner, J.-C., Olteanu, V.-A., Hetherington, A. J., Coupel-Ledru, A., Sun, P., Pridgeon, A. J., et al. (2019). Short- and long-term effects of UVA on Arabidopsis are mediated by a novel cGMP Phosphodiesterase. Curr. Biol. 29, 2580-2585. doi: 10.1016/j.cub.2019.06.071

Jeandroz, S., Wipf, D., Stuehr, D. J., Lamattina, L., Melkonian, M., Tian, Z., et al. (2016). Occurrence, structure, and evolution of nitric oxide synthase-like proteins in the plant kingdom. Sci. Signal. 9:re2. doi: 10.1126/scisignal.aad4403

Ji, Y., Ferracci, G., Warley, A., Ward, M., Leung, K.-Y., Samsuddin, S., et al. (2007). Beta-actin regulates platelet nitric oxide synthase 3 activity through interaction with heat shock protein 90. Proc. Natl. Acad. Sci. U. S. A. 104, 8839-8844. doi: 10.1073/pnas.0611416104

Kawai, J., Kanazawa, M., Suzuki, R., Kikuchi, N., Hayakawa, Y., and Sekimoto, H. (2021). Highly efficient transformation of the model zygnematophycean alga Closterium peracerosum-strigosum-littorale complex by square-pulse electroporation. New Phytol. doi: 10.1111/ nph.17763 [Epub Ahead of Print]

Kone, B. C. (2000). Protein-protein interactions controlling nitric oxide synthases: protein interaction with NOS isoforms. Acta Physiol. Scand. 168, 27-31. doi: 10.1046/j.1365-201x.2000.00629.x

Kumar, A., Castellano, I., Patti, F. P., Palumbo, A., and Buia, M. C. (2015). Nitric oxide in marine photosynthetic organisms. Nitric Oxide 47, 34-39. doi: 10.1016/j.niox.2015.03.001

Kuncewicz, T., Balakrishnan, P., Snuggs, M. B., and Kone, B. C. (2001). Specific association of nitric oxide synthase-2 with Rac isoforms in activated murine macrophages. Am. J. Physiol.-Ren. Physiol. 281, F326-F336. doi: 10.1152/ ajprenal.2001.281.2.F326

León, J., and Costa-Broseta, Á. (2020). Present knowledge and controversies, deficiencies, and misconceptions on nitric oxide synthesis, sensing, and signaling in plants. Plant Cell Environ. 43, 1-15. doi: 10.1111/pce.13617

Mallick, N., Mohn, F.-H., Rai, L., and Soeder, C.-J. (2000). Impact of physiological stresses on nitric oxide formation by green alga, Scenedesmus obliquus. J. Microbiol. Biotechnol. 10, 300-306.

Mallick, N., Rai, L. C., Mohn, F. H., and Soeder, C. J. (1999). Studies on nitric oxide (NO) formation by the green alga Scenedesmus obliquus and the diazotrophic cyanobacterium anabaena Doliolum. Chemosphere 39, 1601-1610. doi: 10.1016/S0045-6535(99)00058-2

Manrique-Gil, I., Sánchez-Vicente, I., Torres-Quezada, I., and Lorenzo, O. (2021). Nitric oxide function during oxygen deprivation in physiological and stress processes. J. Exp. Bot. 72, 904-916. doi: 10.1093/jxb/eraa442

Marchler-Bauer, A., Lu, S., Anderson, J. B., Chitsaz, F., Derbyshire, M. K., DeWeese-Scott, C., et al. (2011). CDD: a conserved domain database for the functional annotation of proteins. Nucleic Acids Res. 39, D225-D229. doi: 10.1093/nar/gkq1189

Martínez-Ruiz, A., Cadenas, S., and Lamas, S. (2011). Nitric oxide signaling: classical, less classical, and nonclassical mechanisms. Free Radic. Biol. Med. 51, 17-29. doi: 10.1016/j.freeradbiomed.2011.04.010

Mazumdar, T., Gorgun, F. M., Sha, Y., Tyryshkin, A., Zeng, S., Hartmann-Petersen, R., et al. (2010). Regulation of NF-KB activity and inducible nitric oxide synthase by regulatory particle non-ATPase subunit 13 (Rpn13). Proc. Natl. Acad. Sci. 107, 13854-13859. doi: 10.1073/pnas.0913495107

Nishiya, T., Matsumoto, K., Maekawa, S., Kajita, E., Horinouchi, T., Fujimuro, M., et al. (2011). Regulation of inducible nitric-oxide synthase by the SPRY domain- and SOCS box-containing proteins. J. Biol. Chem. 286, 9009-9019. doi: $10.1074 /$ jbc.M110.190678

Picciano, A. L., and Crane, B. R. (2019). A nitric oxide synthase-like protein from Synechococcus produces NO/NO3- from l-arginine and NAPDH in a tetrahydrobiopterin- and $\mathrm{Ca}^{2+}$-dependent manner. J. Biol. Chem. 294, 10708-10719. doi: 10.1074/jbc.RA119.008399

Poulos, T. L., Raman, C., and Li, H. (1998). NO news is good news. Structure 6, 255-258. doi: 10.1016/S0969-2126(98)00028-8

Sakihama, Y., Nakamura, S., and Yamasaki, H. (2002). Nitric oxide production mediated by nitrate Reductase in the green alga Chlamydomonas reinhardtii: an alternative NO production pathway in photosynthetic organisms. Plant Cell Physiol. 43, 290-297. doi: 10.1093/pcp/pcf034

Santolini, J. (2019). What does "NO-synthase" stand for. Front. Biosci. 24, 133-171. doi: 10.2741/4711

Santolini, J., André, F., Jeandroz, S., and Wendehenne, D. (2017). Nitric oxide synthase in plants: where do we stand? Nitric Oxide 63, 30-38. doi: 10.1016/j. niox.2016.09.005 
Shen, Q., Zhan, X., Yang, P., Li, J., Chen, J., Tang, B., et al. (2019). Dual activities of plant cGMP-dependent protein kinase and its roles in gibberellin signaling and salt stress. Plant Cell 31, 3073-3091. doi: 10.1105/tpc.19.00510

Stamler, J. S., Lamas, S., and Fang, F. C. (2001). Nitrosylation: The prototypic redox-based signaling mechanism. Cell 106, 675-683. doi: 10.1016/ S0092-8674(01)00495-0

Stuehr, D. J., Santolini, J., Wang, Z.-Q., Wei, C.-C., and Adak, S. (2004). Update on mechanism and catalytic regulation in the NO synthases. J. Biol. Chem. 279, 36167-36170. doi: 10.1074/jbc.R400017200

Tamura, K., Stecher, G., and Kumar, S. (2021). MEGA11: molecular evolutionary genetics analysis version 11. Mol. Biol. Evol. 38, 3022-3027. doi: 10.1093/ molbev/msab120

Tischner, R., Planchet, E., and Kaiser, W. M. (2004). Mitochondrial electron transport as a source for nitric oxide in the unicellular green alga Chlorella sorokiniana. FEBS Lett. 576, 151-155. doi: 10.1016/j.febslet.2004.09.004

Vardi, A., Bidle, K. D., Kwityn, C., Hirsh, D. J., Thompson, S. M., Callow, J. A., et al. (2008). A diatom gene regulating nitric-oxide signaling and susceptibility to diatom-derived aldehydes. Curr. Biol. 18, 895-899. doi: 10.1016/j. cub.2008.05.037

Vázquez-Limón, C., Hoogewijs, D., Vinogradov, S. N., and Arredondo-Peter, R. (2012). The evolution of land plant hemoglobins. Plant Sci. 191, 71-81. doi: 10.1016/j.plantsci.2012.04.013

Venema, R. C., Ju, H., Zou, R., Ryan, J. W., and Venema, V. J. (1997). Subunit interactions of endothelial nitric-oxide synthase: Comparisons to the neuronal and inducible nitric-oxide synthase isoforms. J. Biol. Chem. 272, 1276-1282. doi: $10.1074 /$ jbc.272.2.1276

Weisslocker-Schaetzel, M., André, F., Touazi, N., Foresi, N., Lembrouk, M., Dorlet, P., et al. (2017). The NOS-like protein from the microalgae Ostreococcus tauri is a genuine and ultrafast NO-producing enzyme. Plant Sci. 265, 100-111. doi: 10.1016/j.plantsci.2017.09.019

Wendehenne, D., Lamotte, O., and Pugin, A. (2003). Plant iNOS: conquest of the holy grail. Trends Plant Sci. 8, 465-468. doi: 10.1016/j.tplants.2003.09.003

Winger, J. A., Derbyshire, E. R., Lamers, M. H., Marletta, M. A., and Kuriyan, J. (2008). The crystal structure of the catalytic domain of a eukaryotic guanylate cyclase. BMC Struct. Biol. 8:42. doi: 10.1186/1472-6807-8-42

Zhou, L., and Zhu, D.-Y. (2009). Neuronal nitric oxide synthase: structure, subcellular localization, regulation, and clinical implications. Nitric Oxide 20, 223-230. doi: 10.1016/j.niox.2009.03.001

Conflict of Interest: The authors declare that the research was conducted in the absence of any commercial or financial relationships that could be construed as a potential conflict of interest.

Publisher's Note: All claims expressed in this article are solely those of the authors and do not necessarily represent those of their affiliated organizations, or those of the publisher, the editors and the reviewers. Any product that may be evaluated in this article, or claim that may be made by its manufacturer, is not guaranteed or endorsed by the publisher.

Copyright (c) 2021 Chatelain, Astier, Wendehenne, Rosnoblet and Jeandroz. This is an open-access article distributed under the terms of the Creative Commons Attribution License (CC BY). The use, distribution or reproduction in other forums is permitted, provided the original author(s) and the copyright owner(s) are credited and that the original publication in this journal is cited, in accordance with accepted academic practice. No use, distribution or reproduction is permitted which does not comply with these terms. 